\title{
THE SINGULAR SETS OF AREA MINIMIZING RECTIFI- ABLE CURRENTS WITH CODIMENSION ONE AND OF AREA MINIMIZING FLAT CHAINS MODULO TWO WITH ARBITRARY CODIMENSION'1
}

\author{
BY HERBERT FEDERER
}

Communicated by C. B. Morrey, Jr, February 11, 1970

1. When describing the interior structure of an area minimizing $m$ dimensional locally rectifiable current $T$ in $R^{m+1}$, one calls a point $x \in \operatorname{spt} T \sim \operatorname{spt} \partial T$ regular or singular according to whether or not $x$ has a neighborhood $V$ such that $V \cap$ spt $T$ is a smooth $m$ dimensional submanifold of $\boldsymbol{R}^{m+1}$. As a result of the efforts of many geometers it is known that there exist no singular points in case $m \leqq 6$; a detailed exposition of this theory may be found in [3, Chapter 5]. Recently it was proved in [2] that

$$
Z=\partial\left(E^{8} L R^{8} \cap\left\{x: x_{1}^{2}+x_{2}^{2}+x_{3}^{2}+x_{4}^{2}<x_{3}^{2}+x_{6}^{2}+x_{7}^{2}+x_{8}^{2}\right\}\right)
$$

is a 7 dimensional area minimizing current in $R^{8}$ with the singular point 0 . This implies that, for $m>7, E^{m-7} \times Z$ is an $m$ dimensional area minimizing current in $R^{m-7} \times R^{8} \simeq R^{m+1}$ with the $m-7$ dimensional singular set $R^{m-7} \times\{0\}$. Here we will show (Theorem 1 ) that the Hausdorff dimension of the singular set of an $m$ dimensional area minimizing rectifiable current in $R^{m+1}$ never exceeds $m-7$.

Our method also yields the result (Theorem 2) that the Hausdorff dimension of the singular set of an $m$ dimensional area minimizing flat chain modulo 2 in $R^{m+p}$ never exceeds $m-2$, for arbitrary codimension $p$.

2. We use the terminology of [3]. Given any positive integer $m$ we choose $\Upsilon$ according to $[3,5.4 .7]$ with $n=m+1$ and let

$$
\omega(T)=\left\{x: \Theta^{m}(\|T\|, x) \geqq \Upsilon\right\} \quad \text { for } T \in Q_{m}^{\text {loo }}\left(R^{m+1}\right) .
$$

Whenever $0 \leqq k \in R$ and $A \subset R^{m+1}$ we define $\phi_{\infty}^{k}(A)$ as the infimum of the set of numbers $\sum_{B \in G} a(k) 2^{-k}(\operatorname{diam} B)^{k}$ corresponding to all countable open coverings $G$ of $A$. We see from $[3,2.10 .2]$ that 5304.

AMS Subject Classifications. Primary 2880, 2849; Secondary 2656, 3524, 4640,

Key Words and Phrases. Area minimizing current, rectifiable current, flat chain modulo two, singular point, regular point, Hausdorff dimension, density, oriented tangent cone, Plateau problem.

1 This work was supported in part by a research grant from the National Science Foundation. 


$$
\phi_{\infty}^{k}(A)=0 \text { if and only if } \operatorname{HC}^{k}(A)=0,
$$

and from $[3,2.10 .19(2)]$ that

$$
\Theta^{* k}\left(\phi_{\infty}^{k}\llcorner A, x) \geqq 2^{-k} \text { for } \mathcal{F}^{k} \quad \text { almost all } x \text { in } A .\right.
$$

LEMMA 1. If $Q_{i} \in \mathcal{O}_{m}^{\text {loc }}\left(R^{m+1}\right)$ and $Q_{i}$ is absolutely area minimizing with respect to $R^{m+1}$ for each positive integer $i$,

$$
Q_{i} \rightarrow Q \quad \text { in } \mathfrak{F}_{m}^{100}\left(R^{m+1}\right) \text { as } i \rightarrow \infty,
$$

and $K$ is a compact subset of $R^{m+1} \sim \operatorname{Clos} \bigcup_{i=1}^{\infty}$ spt $\partial Q_{i}$, then

$$
\phi_{\infty}^{k}[\omega(Q) \cap K] \geqq \limsup _{i \rightarrow \infty} \phi_{\infty}^{k}\left[\omega\left(Q_{i}\right) \cap K\right] \text {. }
$$

Proof. We observe that if $V$ is any open set containing $\omega(Q) \cap K$, then $V$ contains $\omega\left(Q_{i}\right) \cap K$ for all sufficiently large integers $i$. Otherwise we could choose a subsequence of points $x_{i} \in \omega\left(Q_{i}\right) \cap K \sim V$ converging to point $x \in K \sim V$. Since

$$
d=\operatorname{dist}\left(K, \bigcup_{i=1}^{\infty} \operatorname{spt} \partial Q_{i}\right)>0,
$$

we would find whenever $d>r>s>0$ that $s^{-m}\left\|Q_{i}\right\| U\left(x_{i}, s\right) \geqq \mathbf{a}(m) \Upsilon$ according to $[3,5.4 .3(3)]$, with $B\left(x_{i}, s\right) \subset U(x, r)$ for large $i$, hence

$$
\|Q\| U(x, r) \geqq \limsup _{i \rightarrow \infty}\left\|Q_{i}\right\| U\left(x_{i}, s\right) \geqq s^{m} \alpha(m) \Upsilon
$$

by $[3,5.4 .2]$. Thus $\|Q\| U(x, r) \geqq r^{m} a(m) \Upsilon$ for $0<r<\delta$, and we could infer that $x \in \omega(Q) \cap(K \sim V)=\varnothing$.

LEMmA 2. If $T \in \mathcal{R}_{m}^{\text {loc }}\left(R^{m+1}\right), T$ is absolutely area minimizing with respect to $R^{m+1}, a \in \mathrm{spt} T \sim \mathrm{spt} \partial T$ and $\Theta{ }^{* k}\left[\phi_{\infty}^{k} L \omega(T), a\right]>0$, then there exists an oriented tangent cone $Q$ of $T$ at a such that $\mathfrak{H C}^{k}[\omega(Q)]>0$.

Proof. Assuming $\Theta^{* k}\left[\phi_{\infty}^{k}\llcorner\omega(T), a]>2{ }^{k} c>0\right.$ and recalling the proof of $[3,5.4 .3]$, in particular the argument on pages 624 and 625 , we choose $\rho_{i}$ and $\beta_{i}$ for each positive integer $i$ so that

$$
\begin{gathered}
0<2 \rho_{i}<i^{-1} \sigma_{i}, \quad \phi_{\infty}^{k}\left[\omega(T) \cap B\left(a, \rho_{i}\right)\right]>\alpha(k) \rho_{i}^{k} 2^{k} c, \\
\beta_{i}^{-1} \in G_{i}, \quad \rho_{i} \leqq\left(1-i 2^{-i}\right) 2 \rho_{i}<\beta_{i}^{-1}<2 \rho_{i} .
\end{gathered}
$$

Then $\phi_{\infty}^{\mathrm{k}}\left[\omega(T) \cap B\left(a, \beta_{i}^{-1}\right)\right]>\boldsymbol{a}(k) \beta_{i}^{-k} c$ and the corresponding currents $Q_{i}=\left(u_{\beta_{i}} \circ \tau_{-a}\right)_{*} T$ satisfy the condition $\phi_{\infty}^{\mathbf{k}}\left[\omega\left(Q_{i}\right) \cap B(0,1)\right]>\boldsymbol{a}(k) c$. A subsequence of $Q_{1}, Q_{2}, Q_{3}, \cdots$ converges in $\mathcal{F}_{m}^{\text {loc }}\left(R^{m+1}\right)$ to an ori- 
ented tangent cone $Q$ of $T$ at $a$, for which $\phi_{\infty}^{k}[\omega(Q) \cap B(0,1)] \geqq a(k) c$ according to Lemma 1.

THEOREM 1. If $T \in \mathcal{R}_{m}^{\mathrm{loc}}\left(R^{m+1}\right), m \geqq 7$ and $T$ is absolutely area minimizing with respect to $R^{m+1}$, then there exists an open set $V$ such that $V \cap$ spt $T$ is an $m$ dimensional submanifold of class $\infty$ of $R^{m+1}$ and

$$
\mathfrak{H C}^{k}\left[R^{m+1} \sim(V \cup \operatorname{spt} \partial T)\right]=0 \text { whenever } m-7<k \in R .
$$

Proof. We use induction with respect to $m$. First we will prove the following statement:

If $M$ is an $\mathfrak{L}^{m+1}$ measurable set, $U$ is an open subset of $R^{m+1}$,

$$
S=\left[\partial ( E ^ { m + 1 } \llcorner M ) ] \left\llcornerU \in \Omega_{m}\left(R^{m+1}\right)\right.\right.
$$

and $S$ is absolutely area minimizing with respect to $R^{m+1}$, then there exist an open set $W$ such that $W \cap$ spt $S$ is an $m$ dimensional submanifold of class $\infty$ of $R^{m+1}$ and

$$
\operatorname{F}^{k}(U \sim W)=0 \text { whenever } m-7<k \in R .
$$

In view of $[3,5.4 .7]$ it suffices to show that

$$
\mathcal{H}^{k}[U \cap \omega(S)]=0 \quad \text { whenever } m-7<k \in R .
$$

Assuming the contrary we choose $k>m-7$ and $a \in U \cap \omega(S)$ so that $\Theta{ }^{* k}\left[\phi_{\infty}^{k}\llcorner\omega(S), a]>0\right.$, apply Lemma 2 to obtain an oriented tangent cone $C$ of $S$ at $a$ with $\mathcal{F}^{k}[\omega(C)]>0$, and infer from $[3,5.4 .3(5),(8)]$ that $C$ is absolutely area minimizing with respect to $R^{m+1}$ and $C$ $=\partial\left(E^{m+1} L N\right)$ for some $\mathscr{L}^{m+1}$ measurable set $N$. Since $\mathcal{H}^{k}\{0\}=0$ we can choose $b \in \omega(C) \sim\{0\}$ so that $\Theta * k\left[\phi_{\infty}^{k}\llcorner\omega(C), b]>0\right.$, and repeat the procedure to construct an oriented tangent cone $D$ of $C$ at $b$ such that ${ }^{C^{k}}[\omega(D)]>0, D$ is absolutely area minimizing with respect to $R^{m+1}$ and $D=\partial\left(E^{m+1}\llcorner P)\right.$ for some $\mathfrak{L}^{m+1}$ measurable set $P$. We infer from $[3,4.3 .16]$ that $D$ is a cylinder with direction $b /|b|$, from $[3,4.3 .15]$ that there exist an isometry $H$ mapping $R \times R^{m}$ onto $R^{m+1}$ and a current $Q \in \mathbb{R}_{m-1}^{\text {loc }}\left(R^{m}\right)$ with $D=H_{\#}\left(E^{1} \times Q\right)$, and from $[3,5.4 .8]$ that $Q$ is absolutely $m-1$ area minimizing with respect to $R^{m}$. We note that $\partial Q=0$ because $\partial D=0$. In case $m \geqq 8$ we inductively obtain an open subset $Y$ of $R^{m}$ such that $Y \cap$ spt $Q$ is an $m-1$ dimensional submanifold of class $\infty$ of $R^{m}$ and $\mathcal{H}^{k-1}\left(R^{m} \sim Y\right)=0$. In case $m=7$ we know from $[3,5.4 .15]$ that spt $Q$ is a 6 dimensional submanifold of class $\infty$ of $R^{7}$, and we take $Y=R^{7}$. In both cases $H(R \times Y) \cap$ spt $D$ is an $m$ dimensional submanifold of class $\infty$ of $R^{m+1}$ and

$$
\mathcal{F}^{k}\left[R^{m+1} \sim H(R \times Y)\right]=\mathcal{H}^{k}\left[R \times\left(R^{m} \sim Y\right)\right]=0
$$


by $[3,2.10 .45]$. Since $D=\partial\left(E^{m+1} L P\right)$ we see that

$$
\Theta^{m}(\|D\|, x)=1 \text { for } x \in H(R \times Y) \cap \operatorname{spt} D,
$$

hence $\omega(D) \subset$ spt $D \sim H(R \times Y)$ and $\Re^{k}[\omega(D)]=0$, which is inconsistent with our previous assertion that $\mathcal{K}^{k}[\omega(D)]>0$.

To deduce the conclusion of the theorem from the statement verified above we suppose $a \in R^{m+1} \sim \operatorname{spt} \partial T$ and proceed as in $[3,5.3 .18]$ to find a positive number $\rho$ and a representation

$$
T L U(a, \rho)=\sum_{i \in Z} S_{i} \text { with }\|T\|\left\llcorner U(a, \rho)=\sum_{i \in Z}\left\|S_{i}\right\|,\right.
$$

where $S_{i}=\left[\partial\left(E^{m+1}\left\llcorner M_{i}\right)\right]\left\llcorner U(a, \rho)\right.\right.$ for certain $\mathscr{L}^{m+1}$ measurable sets $M_{i}$ such that $M_{i} \subset M_{i-1}$; moreover $\left\{i: b \in\right.$ spt $\left.S_{i}\right\}$ is finite whenever $b \in U(a, \rho)$. For each integer $i$ we choose an open set $W_{i}$ such that $W_{i} \cap$ spt $S_{i}$ is an $m$ dimensional submanifold of class $\infty$ of $R^{m+1}$ and

$$
\mathcal{F}^{k}\left[U(a, \rho) \sim W_{i}\right]=0 \text { whenever } m-7<k \in R .
$$

We conclude that $B=U(a, \rho) \sim \bigcup_{i \in Z}$ (spt $\left.S_{i} \sim W_{i}\right)$ is open,

$$
\begin{gathered}
U(a, \rho) \sim B \subset \bigcup_{i \in Z}\left[U(a, \rho) \sim W_{i}\right], \\
\mathcal{C}^{k}[U(a, \rho) \sim B]=0 \quad \text { whenever } m-7<k \in R, \\
B \cap \operatorname{spt} T=\bigcup_{i \in Z} B \cap \operatorname{spt} S_{i}=\bigcup_{i \in Z} B \cap W_{i} \cap \operatorname{spt} S_{i},
\end{gathered}
$$

and $B \cap$ spt $T$ is an $m$ dimensional submanifold of class $\infty$ of $R^{m+1}$ because for each $b \in B \cap$ spt $T$ one can reason as in $[3,5.4 .15$, p. 646] with $a$ replaced by $b$ to see that $\operatorname{Tan}(\operatorname{spt} T, b)$ is an $m$ dimensional vector space, hence infer from $[3,5.3 .18]$ that $b$ is a regular point for $T$.

It is not yet known whether the conclusion of Theorem 1 could be sharpened so as to require that $\mathfrak{H C}^{m-r}(K \sim V)<\infty$ for every compact subset $K$ of spt $T \sim \operatorname{spt} \partial T$; in case $m=7$ this holds according to $[3,5.4 .16]$.

3. Next we discuss area minimizing $m$ dimensional chains with arbitrary codimension $p$ in $R^{m+p}$. When $p>1$ the singular set can have dimension $m-2$, as illustrated in $[3,5.4 .19]$ by the example of holomorphic chains. It follows from $[3,5.3 .16]$ that the singular set of an area minimizing $m$ dimensional rectifiable current $T$ is nowhere dense in spt $T$, but the largest possible value of the dimension of the singular set is not yet known in case $p>1$ and $m>1$.

The situation becomes much simpler when $\boldsymbol{Z}$ is replaced as coeffi- 
cient group by the cyclic group $\boldsymbol{Z}_{2}$ of order 2 . Reducing modulo 2 in the context of geometric measure theory as explained in $[3,4.2 .26]$, one can modify the proof of Theorem 1 to obtain the following proposition:

THEOREM 2. If $T \in \Omega_{m}\left(R^{m+p}\right)$ and $T$ is homologically area minimizing modulo 2 with respect to $R^{m+p}$, which means that $M(T+\partial S+2 R)$ $\geqq M(T)$ whenever $S \in \mathbb{R}_{m+1}\left(R^{m+p}\right)$ and $R \in \Omega_{m}\left(R^{m+p}\right)$, then there exists an open set $V$ such that $V \cap$ spt $T$ is an $m$ dimensional submanifold of class $\infty$ of $\boldsymbol{R}^{m+p}$ and

$\mathfrak{F}^{k}\left[R^{m+p} \sim\left(V \cup \operatorname{spt}^{2} \partial T\right)\right]=0 \quad$ whenever $\sup \{m-2,0\}<k \in R$.

In fact the extension of our two lemmas from $R^{m+1}$ to $R^{m+p}$ is trivial, the present current $T$ is representative modulo 2 , hence $\omega(T) \sim$ spt $^{2} \partial T$ equals the singular subset of spt $T \sim$ spt $^{2} \partial T$, and the induction now starts with the case $m=1$ where the singular set is known to be empty.

For $m=2$ it was found in $[1$, Theorem $3(1)]$ that the singular set is isolated and spt $T \sim$ spt $^{2} \partial T$ is the image of an immersion of a 2 dimension manifold in $R^{2+p}$. However, for $m>2$ it is not yet known whether one could sharpen the conclusion of Theorem 2 so as to require that $3^{m-2}(K \sim V)<\infty$ for every compact subset $K$ of spt $T \sim \operatorname{spt}^{2} \partial T$.

Recalling $[3,5.4 .4]$ one sees that Theorem 2 remains valid with $R^{m+p}$ replaced by any $m+p$ dimensional Riemmanian manifold of class $\infty$.

For the study of interior regularity of solutions of the problem of least area, use of $m$ dimensional flat chains modulo 2 is substantially equivalent to use of sets with finite $m$ dimensional Hausdorff measure as employed in Reifenberg's approach presented in [4, Chapter 10], provided $G=Z_{2}$ and $L$ is cyclic (see $[4$, p. 411]). Then our method shows that the Hausdorff dimension of the singular set of Reifenberg's solution of the $m$ dimensional Plateau problem does not exceed $m-2$.

\section{REFERENCES}

1. F. J. Almgren, Jr., Some interior regularity theorems for minimal surfaces and an extension of Bernstein's theorem, Ann. of Math. (2) 84 (1966), 277-292. MR 34 \#702.

2. E. Bombieri, E. DeGiorgi and E. Giusti, Minimal cones and the Bernstein theorem, Invent. Math. 7 (1969), 243-269.

3. H. Federer, Geometric measure theory, Die Grundlehren der math. Wissenschaften, Band 153, Springer-Verlag, Berlin and New York, 1969.

4. C. B. Morrey, Jr., Multiple integrals in the calculus of variations, Die Grundlehren der math. Wissenschaften, Band 130, Springer-Verlag, Berlin and New York, 1966. MR 34 \#2380.

Brown University, Providence, Rhode Island 02912 